\title{
Evaluation of the efficacy of unipolar and bipolar spinal dorsal root ganglion radiofrequency thermocoagulation in the treatment of postherpetic neuralgia
}

\author{
Jianjun Zhu*, Ge Luo*, Qiuli He, and Ming Yao \\ Department of Anesthesiology and Pain Research Center, The First Hospital of Jiaxing or The Affiliated Hospital of Jiaxing University, Jiaxing, China
}

Received September 11, 2021

Revised October 28, 2021

Accepted November 12, 2021

Handling Editor: Younghoon Jeon

\section{Correspondence}

Ming Yao

Department of Anesthesiology and Pain

Research Center, The First Hospital

of Jiaxing or The Affiliated Hospital

of Jiaxing University, No. 1882 South

Zhonghuan Road, Nanhu District, Jiaxing

314001, Zhejiang, China

Tel: +86-13456218632

Fax: +86-0573-82519999

E-mail: jxyaoming666@163.com

*These authors contributed equally to this work.

\begin{abstract}
Background: Different views have been proposed on the radiofrequency treatment modes and parameters of radiofrequency thermocoagulation of the spinal dorsal root ganglion for the treatment of postherpetic neuralgia (PHN). It is urgent to identify a more effective therapy for patients with PHN.

Methods: Patients who underwent radiofrequency thermocoagulation therapy for PHN were retrospectively reviewed and were divided into a radiofrequency thermocoagulation (CRF) and double neddles radiofrequency thermocoagulation (DCRF). The pain scores (numerical rating scale, NRS) were evaluated the following time points: before the operation, 1 day, 3 months, 6 months, 1 year, and 2 years after operation. The incidence of complications and the degree of pain relief were evaluated. The in vitro ovalbumin experiment was used to indicate the effects of radiofrequency thermocoagulation.

Results: Compared with the preoperative NRS scores, the postoperative NRS scores decreased significantly; the NRS scores of the DCRF group was lower than that of the CRF group at all time points from 6 months to 2 years following the operation. The total effective rate of the DCRF group was significantly higher than that of the CRF group at 2 years following the operation. The incidence of numbness in the DCRF group was higher than that noted in the CRF group. The ovalbumin experiments in vitro indicated that the effects of radiofrequency thermocoagulation were optimal when the distance between the two needles was $5 \mathrm{~mm}$.

Conclusions: DCRF with a $5 \mathrm{~mm}$ spacing exhibits a longer duration and higher effective rate in the treatment of PHN and is worth promoting.
\end{abstract}

Key Words: Chronic Pain; Electrocoagulation; Ganglia, Spinal; Herpes Zoster; In Vitro Techniques; Incidence; Needles; Neuralgia, Postherpetic; Ovalbumin; Pain Management; Radiofrequency Therapy; Spine.

\section{INTRODUCTION}

Herpes zoster is caused by the varicella-zoster virus, which infects the dorsal root ganglion (DRG) or cerebral nerve $[1,2]$. It is common in middle-aged and elderly people with impaired immunity. The main risk factors include woman gender, family history, and autoimmune diseases [3]. Although the herpes zoster vaccine has been widely used in certain countries $[2,4,5]$, recent studies have shown that the prevalence rate of this virus continues to rise and that (a) This is an open-access article distributed under the terms of the Creative Commons Attribution Non-Commercial License (http://creativecommons.org/licenses/by-nc/4.0/), which permits unrestricted non-commercial use, distribution, and reproduction in any medium, provided the original work is properly cited.

(C) The Korean Pain Society, 2022
Author contributions: Jianjun Zhu: Investigation; Ge Luo: Formal analysis; Qiuli He: Data curation; Ming Yao: Project administration. 
the age of the disease exhibits a downward trend $[6,7]$. When the cellular immune function of the host is low, the virus is activated again, causing inflammation and necrosis of the ganglia. Concomitantly, the activated virus can move along the peripheral nerve fibers to the skin to develop herpes, which is associated with pain [1]. Its severity usually depends on the presence of pain before the formation of the rash, the severity of the rash, sex, age, and impaired immune function [8-10].

Radiofrequency therapy of the spinal DRG is considered to be one of the most effective intervention methods for the treatment of postherpetic neuralgia (PHN) due to its small trauma, effective control, high safety, optimal accuracy, and reliable repeatability. This method has been widely used previously $[11,12]$. Pulsed radio frequency (PRF) is considered to regulate only the nerve and therefore causes almost no nerve damage [13]. Radiofrequency thermocoagulation is considered to cause nerve damage, resulting in side effects, such as hypoesthesia and decreased muscle strength [13]. However, the effective rate of PRF alone in the clinical treatment is low and its effective time is short [14].

Unipolar radiofrequency means that a single radio frequency puncture needle reaches the operation site, and the other electrode is attached to the body surface, thus forming a large, closed loop. When the radio frequency needle encounters the impedance of human tissue, it produces heat energy and achieves the effect of radiofrequency thermocoagulation. Bipolar radio frequency means that two radio frequency puncture needles reach the operation site and form a small, closed loop in the operation area. According to the impedance of human tissue between the two needles, heat energy is generated to achieve the effect of radiofrequency thermocoagulation. The main difference between the two patterns is that in the unipolar radiofrequency the current passes through the skin tissue where it is lost mainly in the form of radiation heating; while in the bipolar radiofrequency, the current is relatively more concentrated between the needles in the form of linear heating.

Therefore, it is urgent to seek a newer and more effective radiofrequency therapy for patients with PHN. The purpose of this study was to compare the effective rate, effective maintenance time and complications of bipolar and unipolar radiofrequency thermocoagulation of the spinal DRG in the treatment of PHN, so as to guide clinical practice.

\section{MATERIALS AND METHODS}

\section{Clinical data}

A total of 60 patients that underwent radiofrequency thermocoagulation therapy for PHN in the Pain Department of the Affiliated Houspital of Jiaxing University from January 2013 to November 2017 were retrospectively reviewed. Among them, 34 males and 26 females were present, aged from 49 to 93 years, with an average age of $72.5 \pm 9.4$ years. Radiofrequency thermocoagulation of the spinal DRG was performed by the same doctor with sufficient clinical pain management experience. All patients signed an informed consent form, and our study was conducted in accordance with the Declaration of Helsinki. Moreover, this study was examined and approved by the Ethics Committee of the First Hospital of Jiaxing (LS-2010-119).

\section{Inclusion and exclusion criteria}

1) The following inclusion criteria were used: (1) The subjects met the clinical diagnostic criteria of post-herpetic neuralgia; (2) The age of the subjects was higher than and/ or equal to 18 years; (3) The diseased region of the herpes zoster only involved the unilateral trunk nerve, that is, the thoracic 1-12 nerve; (4) The subjects' numerical rating scale (NRS) score was higher than 4 when they were admitted to the hospital; (5) The course of the herpes zoster disease was $\geq 3$ months; (6) The subjects underwent radiofrequency thermocoagulation (CRF) or double neddles radiofrequency thermocoagulation (DCRF); (7) All the subjects agreed and received follow-up by telephone.

2) The following exclusion criteria were used: (1) Patients with a history of malignant tumors; (2) Patients that had received immunosuppressive therapy; (3) Uncooperative patients with mental illness, mental retardation, or confusion; (4) Patients with severe liver, kidney, heart, and lung diseases; (5) Patients that had received a history of invasive treatment prior to operation; (6) Patients that withdrew from the operation due to lack of cooperation with the surgeon during the procedure; (7) Patients that had received other invasive treatment or other surgeries following the operation; (8) Patients with herpes zoster at the lumbar segmental level; (9) Patients that lacked basic information and could not be followed up following the operation.

The patients were selected according to the inclusion and exclusion criteria and all patients were divided into the bipolar group (DCRF group, $n=24$ ) and the unipolar group (CRF group, $\mathrm{n}=36$ ) by reviewing their intraoperative imaging data. 

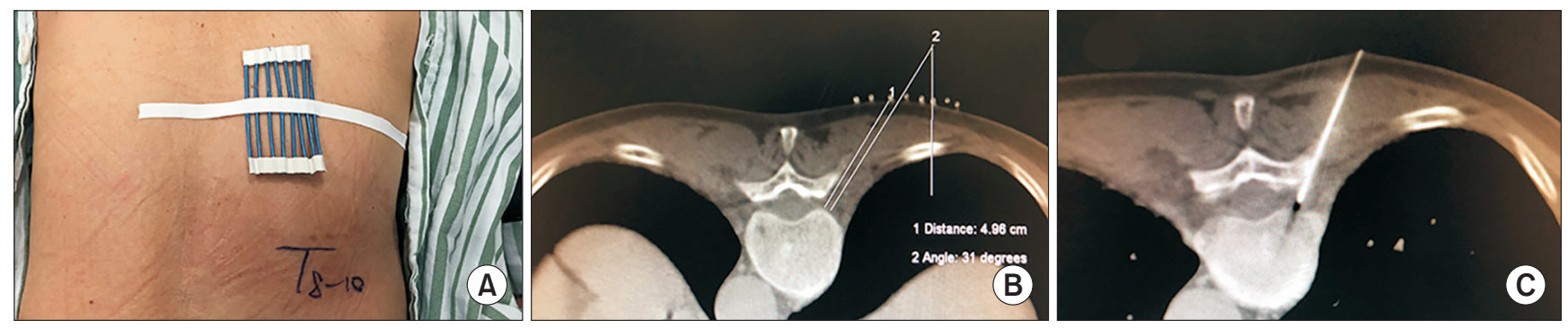

Fig. 1. Surgical procedure of radiofrequency thermocoagulation. (A) Place positioning mark. (B) Design the puncture route. (C) Puncture to predetermined position.
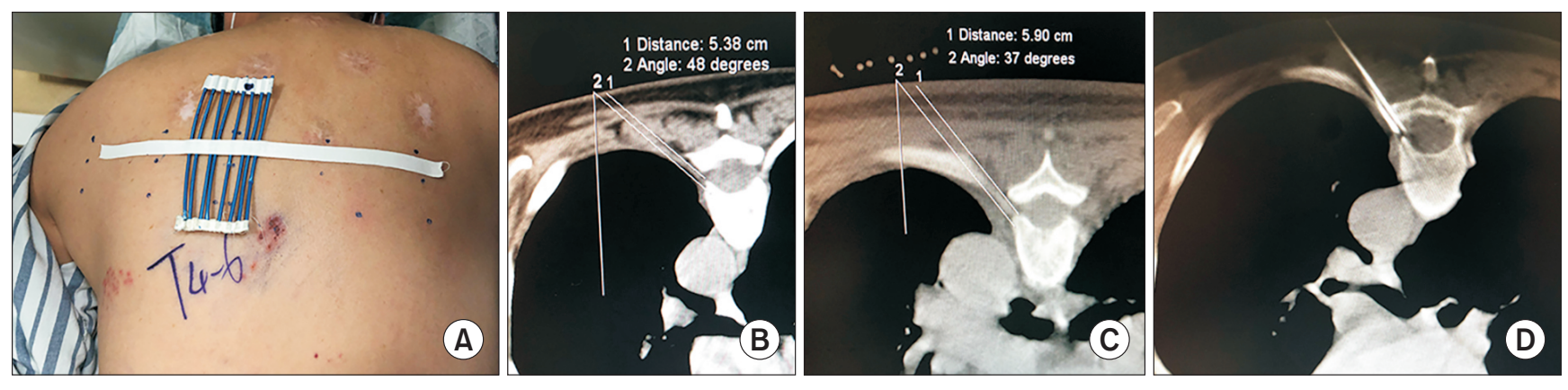

Fig. 2. Surgical procedure of double neddles radiofrequency thermocoagulation. (A) Place positioning mark. (B, C) Design the puncture route. (D) Puncture to predetermined position.

\section{Surgical methods}

The patients entered the computed tomography (CT) treatment room and laid in a prone position on the treatment bed. A positioning grid was placed at the patient's preoperative mark and fixed with adhesive tape (Fig. 1A, 2A). Then, with the help of CT software, the puncture target was pre-designed, and the puncture depth and angle were calculated (Fig. 1B, 2B, 2C). After that, local infiltration of anesthesia was performed with $2 \%$ lidocaine (Hubei Tianyao Pharmaceutical Co., Ltd., Xiangyang, Hubei, China) at a volume of $0.5 \mathrm{~mL}$. Under the guidance of CT (Siemens SomatomEmotion system - Siemens HealthCare, Malvern, PA), the puncture needle (Inomed Health Ltd, Hopwood Lane, Halifax, Germany) was used to create a puncture following the preset angle and the path to the specified position. In the CRF group, a single puncture needle was used to puncture a pre-designed target (Fig. 1C). In the DCRF group, two puncture needles were inserted from different angles towards the pre-designed target (Fig. 2D).

Following completion of the initial puncture, a CT scan was performed again to confirm the needle placement. Further adjustment of the direction of the needle was performed to the outlet of the nerve root at the ventral superior edge of the intervertebral foramen in the affected area. Then the radiofrequency $(\mathrm{RF})$ device (Baylis Medical Company Inc., Toronto, ON, Canada) was connected and the following tests were performed: (1) The sensory stimulation was carried out under $50 \mathrm{~Hz}$ and $0.1 \mathrm{~ms}$ and the current threshold inducing abnormal pain sensation was observed to judge the accuracy of the puncture site and record the current $(\mathrm{mA})$ at this time. (2) The motor stimulation was carried out under $2 \mathrm{~Hz}$ and $1.0 \mathrm{~ms}$ in order to observe the current threshold that could make the muscle beat in the area, which was innervated by the DRG, and to record the current ( $\mathrm{mA}$ ) at the same time. Subsequently, the tip position was adjusted again until the movement and sensation areas were completely covered, without affecting the normal area. Finally, the radio frequency parameters were set. The parameters of the patients in the CRF and DCRF groups were both $95^{\circ} \mathrm{C}$ and 300 seconds $[15,16]$. During the operation, patients didn't receive any additional blocks, the reaction of the patients was observed, and the incidence of the complications was assessed. A record from all the patients was made.

\section{Observation and follow-up}

The preoperative data included age, sex, pain course, pain location, and preoperative pain NRS score [17], as well as the NRS score 1 day following the operation. The postoperative data included the NRS score, pain relief, operative complications, and side effects at 3 months, 6 months, 1 year, and 2 years, all of which were evaluated by telephone 

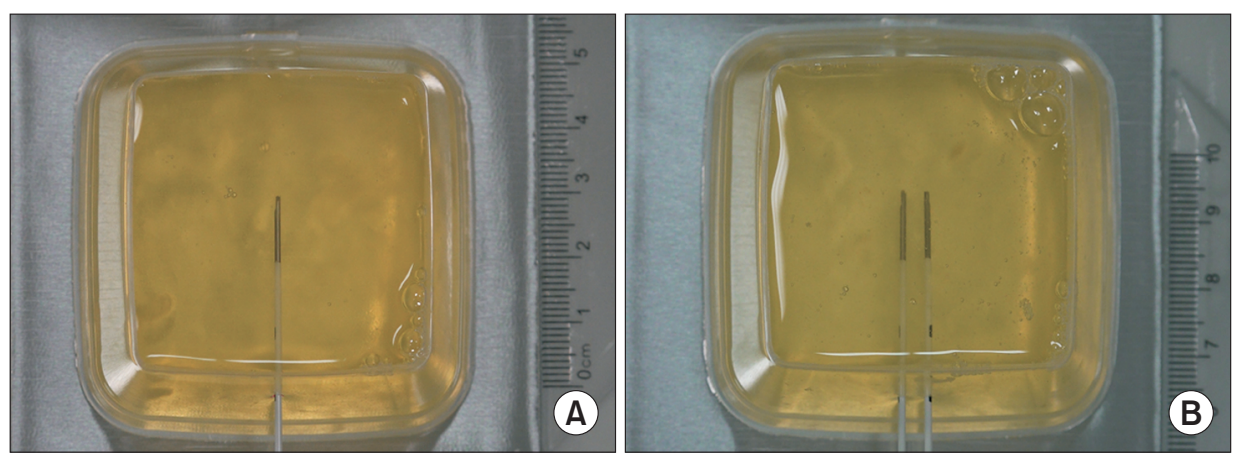

Fig. 3. The active tip of the needle was wrapped by ovalbumin in CRF group (A) and DCRF (B). CRF: radiofrequency thermocoagulation, DCRF: double neddles radiofrequency thermocoagulation.

follow-up. The medical staff in the non-operation group followed up the patients by telephone.

Pain was measured with a pain NRS score, ranging from 0 (no pain) to 10 (the most unbearable pain). The degree of pain relief was evaluated according to the curative effect of the patient 2 years following the operation. The curative effect was divided into 4 grades (a, b, c, d) according to the ratio of (the difference between preoperative NRS and postoperative NRS scores)/preoperative NRS score $\times 100 \%$. The following grades were defined as follows $[18,19]$ : (a) complete relief (CR), pain relief more than $75 \%$; (b) partial relief (PR), pain relief 50 to $75 \%$; (c) mild relief (MR), pain relief 25 to $50 \%$; and (d) unresponsive relief, pain relief less than $25 \%$. The total effective rate (\%) was defined by the following equation: $[(\mathrm{CR}+\mathrm{PR}+\mathrm{MR}) / \mathrm{n}] \times 100 \%$ and the marked rate $(\%)=[(\mathrm{CR}+\mathrm{PR}) / \mathrm{n}] \times 100 \%$.

In addition, the incidence of the complications, including short-term pneumothorax, such as hematoma, infection and spinal cord injury, and the long-term complications, such as numbness in the corresponding innervated areas and abdominal dilatation were recorded. Moreover, the incidence of numbness and abdominal distension was calculated in the affected area.

\section{Power of the study}

The analysis of the clinical data of the patients suggested that the marked rate of 2 years after the surgery was $53.33 \%$ in the CRF group and $94.74 \%$ in the DCRF group. The power of the study was estimated at $90 \%$, with a $95 \%$ confidence interval and a 2 -sided type I error of $5 \%$. Therefore, the present study required 19 patients in each treatment arm. To compensate for patients who may have been lost to follow-up, 60 patients were reviewed for the two groups. Among them, 36 patients were included in the CRF group and 24 patients in the DCRF group.

\section{Ovalbumin in vitro test}

In the in vitro ovalbumin test, the puncture needle and ra- dio frequency electrode used in the clinic were completely immersed in the culture medium, so that the active tip of the needle was wrapped by ovalbumin (Fig. 3). In the CRF group and DCRF group, the electrodes were placed in parallel and connected to the output socket of the radio frequency generator. However, in the DCRF group, the distance between the two electrodes was gradually adjusted according to the experimental requirements. When the radio frequency generator was operating, the tip temperature was kept at the target temperature of $95^{\circ} \mathrm{C}$. The image of the thermal coagulation of ovalbumin was obtained directly on the side and directly above the active tip of the puncture needle. A measuring ruler was placed for reference (Subsidiary Video 1, 2). In the DCRF group, the distance between the two electrodes was set at 2, 3, 4, 5, 6,7 , and $8 \mathrm{~mm}$. The length, width, height, and final weight of the thermal coagulation in the CRF and DCRF groups were recorded by manual measurement of thermal coagulation at different intervals and different time points (Fig. 4). Therefore, the effects of the bipolar distance and heating time on the size of coagulation were analyzed further.

\section{Statistical analysis}

The SPSS ver. 25.0 (IBM Co., Armonk, NY) was used for statistical analysis. The frequency was used as the main variable for the classification of the groups. The normality of the distribution was tested by the Kolmogorov-Smirnov test. The variables of the non-normal distribution were presented by the median (quartile spacing) and were compared by the Mann-Whitney $U$-test. The variables of the normal distribution were analyzed and compared by oneway analysis of variance, and the values were expressed as mean \pm standard deviation. Multiple comparisons were made using the Bonferroni correction and after adjusting the significance, a corrected $\alpha$ of 0.0033 was obtained. The analysis was performed using the Pearson's chi-squared on the pain relief degree. A bilateral $P<0.05$ indicated that the differences were statistically significant. 

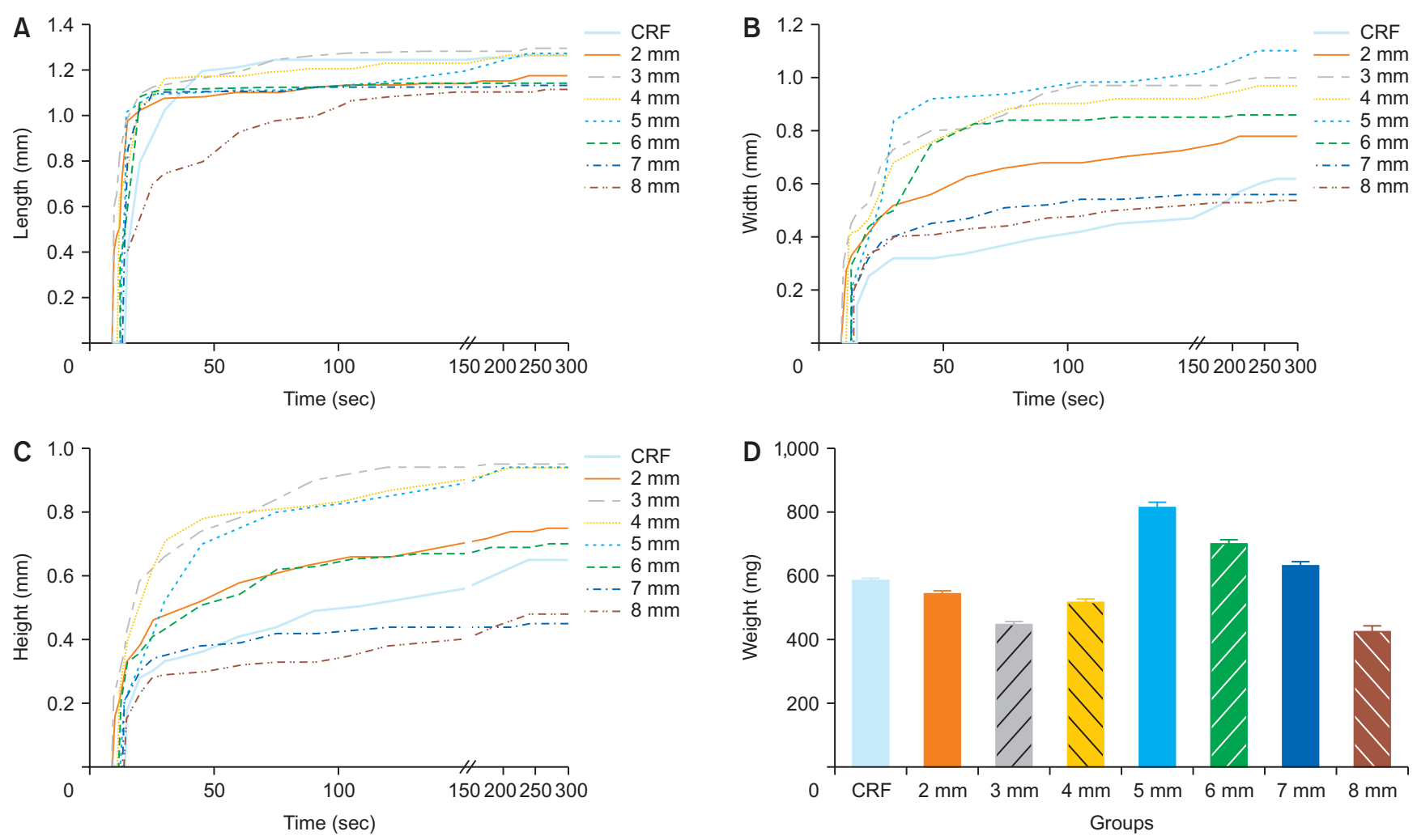

Fig. 4. The length (A), width (B), height $(C)$ and weight (D) of coagulum in each group. CRF: radiofrequency thermocoagulation.

\section{RESULTS}

\section{Analysis of clinical characteristics of patients}

The comparison of the basic demographic and clinical data of the two groups prior to the operation indicated no significant differences between the two groups in terms of sex, age, course of pain, location of pain and, NRS score prior to the operation (Table 1).

\section{Assessment of the success of surgical puncture}

The results of the electrophysiological test indicated that the sensory test of the CRF group was $0.39 \pm 0.12(\mathrm{~mA})$, whereas the DCRF group was $0.36 \pm 0.11(\mathrm{~mA}), P=0.390$; The exercise test of the CRF group was $0.41 \pm 0.14(\mathrm{~mA})$ and the DCRF group was $0.34 \pm 0.11(\mathrm{~mA}), P=0.071$. The difference was not significant (Table 2).

\section{Pain scores (NRS)}

1) Comparative analysis of NRS scores prior to and following operation in the CRF group

The preoperative NRS scores and the postoperative scores at 1 day, 3 months, 6 months, 1 year, and 2 years in the CRF group are shown in Table 3. The postoperative NRS score
Table 1. Clinical characteristics of patients

\begin{tabular}{lccc}
\hline \multicolumn{1}{c}{ Variable } & CRF & DCRF & $P$ value \\
\hline Age & $70.8 \pm 10.2$ & $72.7 \pm 8.0$ & 0.147 \\
Sex & 17 & 11 & 0.933 \\
$\quad$ Male & 13 & 8 & \\
$\quad$ Female & & & \\
Incidence of painful area (\%) & 1 & 0 & \\
T1 & 3 & 4 & \\
T2 & 5 & 6 & \\
T3 & 5 & 7 & \\
T4 & 11 & 4 & \\
T5 & 14 & 4 & \\
T6 & 17 & 3 & \\
T7 & 14 & 6 & \\
T8 & 8 & 3 & \\
T9 & 5 & 7 & \\
T10 & 3 & 6 & \\
T11 & 2 & 6 & \\
T12 & $10.7 \pm 17.5$ & $8.6 \pm 6.8$ & 0.228 \\
The length of painful time (mo) & $5.5 \pm 1.0$ & $5.3 \pm 0.7$ & 0.083 \\
NRS score before surgery & & & \\
\hline
\end{tabular}

Values are presented as mean \pm standard deviation or number only. CRF: radiofrequency thermocoagulation, DCRF: double neddles radiofrequency thermocoagulation, NRS: numerical rating scale.

at each time point was lower than that prior to the operation. The difference was significant (Table 3).

https://doi.org/10.3344/kjp.2022.35.1.114 
2) Comparative analysis of NRS scores prior to and following operation in the DCRF group

The preoperative NRS scores, and scores at postoperative day 1, 3 months, 6 months, 1 year, and 2 years in the DCRF group are shown in Table 3 . The postoperative NRS score at each time point was lower than that prior to the operation and the difference was statistically significant (Table 3).

Table 2. Electrophysiological results of the CRF group and the DCRF group

\begin{tabular}{lcc}
\hline Group & Sensory test $(\mathrm{mA})$ & Exercise test $(\mathrm{mA})$ \\
\hline CRF & $0.39 \pm 0.12$ & $0.41 \pm 0.14$ \\
DCRF & $0.36 \pm 0.11$ & $0.34 \pm 0.11$ \\
$P$ value & 0.39 & 0.07 \\
\hline
\end{tabular}

Values are presented as mean \pm standard deviation.

CRF: radiofrequency thermocoagulation, DCRF: double neddles radiofrequency thermocoagulation.
3) Comparative analysis of NRS scores prior to and following operation between the CRF and DCRF groups

No significant differences were noted in the NRS score between the DCRF and CRF groups prior to the operation and at day 1 and 3 months following the operation. However, at 6 months following the operation, the NRS score of the DCRF group was significantly lower than that of the CRF group. This effect was noted for the first time. Subsequently, at all the time points from 6 months to 2 years following the operation, the NRS score of the DCRF group was significantly lower than that of the CRF group (Table 3).

\section{Pain relief degree}

From the follow-up, at 2 years following the operation, the marked rate of the CRF group (53.33\%) was significantly lower than that of the DCRF group (94.74\%). This was our primary outcome. No significant differences were noted in the marked rate at other time points. The total effective rate of the CRF and DCRF groups at each time point fol-

Table 3. Analysis of numerical rating scale before and after surgery in different treatment groups

\begin{tabular}{|c|c|c|c|c|c|c|}
\hline \multirow{2}{*}{ Group } & \multirow{2}{*}{ Before surgery } & \multicolumn{5}{|c|}{ Time after surgery } \\
\hline & & 1 day & $3 \mathrm{mo}$ & $6 \mathrm{mo}$ & $1 \mathrm{yr}$ & $2 \mathrm{yr}$ \\
\hline \multicolumn{7}{|c|}{ Comparison within groups } \\
\hline CRF & $5.3 \pm 0.7$ & $1.8 \pm 0.6$ & $2.5 \pm 1.3$ & $2.6 \pm 1.3$ & $2.7 \pm 1.2$ & $2.6 \pm 1.1$ \\
\hline$P$ value & & 0.001 & 0.001 & 0.001 & 0.001 & 0.001 \\
\hline DCRF & $5.5 \pm 1.0$ & $2.0 \pm 0.5$ & $2.2 \pm 0.8$ & $2.1 \pm 0.9$ & $2.2 \pm 1.0$ & $2.0 \pm 0.8$ \\
\hline$P$ value & & 0.001 & 0.001 & 0.001 & 0.001 & 0.001 \\
\hline \multicolumn{7}{|c|}{ Comparison between groups } \\
\hline CRF & $5.3 \pm 0.7$ & $1.8 \pm 0.6$ & $2.5 \pm 1.3$ & $2.6 \pm 1.3$ & $2.7 \pm 1.2$ & $2.6 \pm 1.1$ \\
\hline DCRF & $5.5 \pm 1.0$ & $2.0 \pm 0.5$ & $2.2 \pm 0.8$ & $2.1 \pm 0.9$ & $2.12 \pm 1.0$ & $2.0 \pm 0.8$ \\
\hline$P$ value & 0.401 & 0.522 & 0.254 & 0.024 & 0.041 & 0.050 \\
\hline
\end{tabular}

Values are presented as mean \pm standard deviation.

CRF: radiofrequency thermocoagulation, DCRF: double neddles radiofrequency thermocoagulation.

Table 4. Analysis of pain relief degree

\begin{tabular}{|c|c|c|c|c|c|c|c|c|}
\hline \multirow{2}{*}{ Degree } & \multicolumn{2}{|c|}{ Postoperative 3 mo } & \multicolumn{2}{|c|}{ Postoperative 6 mo } & \multicolumn{2}{|c|}{ Postoperative $1 \mathrm{yr}$} & \multicolumn{2}{|c|}{ Postoperative $2 \mathrm{yr}$} \\
\hline & CRF & DCRF & CRF & DCRF & CRF & DCRF & CRF & DCRF \\
\hline CR & 8 & 4 & 7 & 6 & 4 & 6 & 3 & 7 \\
\hline PR & 10 & 12 & 11 & 10 & 15 & 10 & 13 & 11 \\
\hline MR & 9 & 3 & 8 & 3 & 8 & 2 & 10 & 1 \\
\hline NR & 3 & 0 & 4 & 0 & 3 & 1 & 4 & 0 \\
\hline The marked rate (\%) & 60.00 & 84.21 & 60.00 & 84.21 & 63.33 & 84.21 & 53.33 & 94.74 \\
\hline$P$ value & 0.073 & & 0.073 & & 0.115 & & 0.002 & \\
\hline The total effective rate (\%) & 90.00 & 100 & 86.67 & 100 & 90.00 & 94.74 & 86.67 & 100 \\
\hline$P$ value & 0.273 & & 0.148 & & 0.999 & & 0.148 & \\
\hline
\end{tabular}

The marked rate $(\%)=[(C R+P R) / n] \times 100 \%$. The total effective rate $(\%)=[(C R+P R+M R) / n] \times 100 \%$.

CRF: radiofrequency thermocoagulation, DCRF: double neddles radiofrequency thermocoagulation, CR: complete relief, PR: partial relief, MR: mild relief, NR: unresponsive relief. 
Table 5. Comparisons of long-term postoperative complications between CRF and DCRF

\begin{tabular}{|c|c|c|c|c|c|c|c|c|}
\hline \multirow{2}{*}{ Complication } & \multicolumn{2}{|c|}{ Postoperative 3 mo } & \multicolumn{2}{|c|}{ Postoperative 6 mo } & \multicolumn{2}{|c|}{ Postoperative $1 \mathrm{yr}$} & \multicolumn{2}{|c|}{ Postoperative 2 yr } \\
\hline & CRF & DCRF & CRF & DCRF & CRF & DCRF & CRF & DCRF \\
\hline \multicolumn{9}{|l|}{ Numbness } \\
\hline Yes & 19 & 17 & 18 & 17 & 16 & 16 & 8 & 13 \\
\hline No & 11 & 2 & 12 & 2 & 14 & 3 & 22 & 6 \\
\hline Incidence rate (\%) & 63.33 & 89.48 & 60.00 & 89.48 & 53.33 & 84.21 & 26.67 & 68.42 \\
\hline$P$ value & \multicolumn{2}{|c|}{0.043} & \multicolumn{2}{|c|}{0.026} & \multicolumn{2}{|c|}{0.027} & \multicolumn{2}{|c|}{0.004} \\
\hline Abdominal distension & & & & & & & & \\
\hline Yes & 21 & 12 & 16 & 9 & 13 & 7 & 9 & 6 \\
\hline No & 9 & 7 & 14 & 10 & 17 & 12 & 21 & 13 \\
\hline Incidence rate (\%) & 70.00 & 63.33 & 53.33 & 47.37 & 43.33 & 36.84 & 30.00 & 31.58 \\
\hline$P$ value & \multicolumn{2}{|c|}{0.619} & \multicolumn{2}{|c|}{0.684} & \multicolumn{2}{|c|}{0.652} & \multicolumn{2}{|c|}{0.907} \\
\hline
\end{tabular}

lowing the operation is shown in Table 4. No significant differences were noted between the two groups (Table 4).

\section{Operative complications}

The main operative complications were pneumothorax, spinal cord injury, hematoma, infection, numbness, and abdominal distension. The review of the disease history of the patients demonstrated no intraoperative complications in the aforementioned two groups.

No short-term postoperative complications were noted in both groups. However, observation of long-term postoperative complications demonstrated that all patients exhibited varying degrees of skin numbness in the corresponding innervation area, whereas certain patients had abdominal distension. No other serious or permanent complications and side effects were observed during the follow-up period. The statistical results of the incidence of long-term postoperative complications are shown in Table 5. Significant differences were noted in the incidence of numbness at each time point between the two groups, whereas no significant difference was noted in the incidence of abdominal distension between the two groups (Table 5).

\section{Analysis of ovalbumin in vitro test}

1) The length, width, and height of coagulation at different time points during the ovalbumin in vitro test in each group were measured as shown in Fig. 4A-C. The results of the length measurement indicated no difference in the final length of each group, while the width and height of each group indicated that the width and height of the 5 mm group was the widest and highest (Fig. 4A-C).

2) The final coagulant weight of each group in the oval- bumin in vitro test is shown in Fig. 4D. When the distance of the two electrodes was $5 \mathrm{~mm}$, the thermal solidification effect was the best and the weight of the coagulum was the highest, reaching $818.57 \pm 26.02 \mathrm{mg}$ (Fig. 4D).

Based on the above experimental results of the ovalbumin in vitro test, it is inferred that when the distance of the two electrodes was $5 \mathrm{~mm}$, the effects of the radiofrequency thermocoagulation were optimal. This finding has instructive significance for radiofrequency thermocoagulation of the bipolar spinal DRG in the treatment of PHN.

\section{DISCUSSION}

In recent years, radiofrequency therapy has been widely recognized due to its high success rate, optimal efficacy, high safety, and high patient satisfaction. It has gradually become one of the mainstream methods for the treatment of neuralgia. Previous research studies on its mechanism have been carried out gradually.

Previous studies have shown that PRF can regulate the synaptic plasticity of nerve cells through in vivo experiments and have speculated that the analgesic effect of PRF may be achieved through neuro-regulation [13]. CRF produces high-frequency current, induces ion oscillation in tissues, heats up tissues, increases local temperature, and inactivates hypersensitive nerve endings caused by pathological changes through thermal effect, so as to achieve the purpose of the treatment. At present, it is believed that the analgesic effect of PRF may be due to the temporary blocking of neural signals by neurons through the nerve conduction pathway, while the analgesic effect of CRF may be through the permanent blocking of neural signals through the neural pathway [13].

At present, in the clinic, radio frequency current is used 
to treat neuralgia [14] and has been used extensively, such as in cases of paroxysmal collective headache [20], primary trigeminal neuralgia [15,21-23], glossopharyngeal neuralgia [24], PHN [11,12,25,26], chronic knee pain [27], and postherpetic trigeminal neuralgia [28]. In addition, certain studies on the use of different radio frequency modes for the treatment of neuralgia have been carried out consecutively. Previous studies have shown that the effect of simple pulsed radiofrequency is not successful, whereas the therapeutic effect of pulsed radiofrequency combined with radiofrequency thermocoagulation is suitable for its clinical use [22,23]. In the comparative study of unipolar and bipolar groups, it has been found that the bipolar group exhibited more advantages in pain relief and functional recovery $[15,27]$.

In the clinical treatment of PHN, discussion and research on unipolar and bipolar radiofrequency have not been reported. In the present study, an electrophysiological test was used to evaluate whether the puncture was in the correct place for clinical applications. The data demonstrated that when the electrophysiological test results were within $1.0 \mathrm{~mA}$, the puncture needle was close to the target ganglion, suggesting that the puncture was in the correct place.

Moreover, the incidence of numbness in the DCRF group was higher than that in the CRF group at each time point. It is interesting to note that certain patients had abdominal distension following the operation. Therefore, we conducted follow-up statistics on this initial result. The results indicated no significant differences in the incidence of abdominal distension between the two groups. However, the association between this phenomenon and the procedure's curative effects requires further investigation.

Previous studies have suggested that the extension of the time of radiofrequency exposure is not an effective method for the treatment of neuropathic pain and excessive exposure is associated with increased neuronal damage $[13,29]$. In order to assess the optimal thermal coagulation effect in the bipolar procedure more clearly, we explored the radio frequency duration and bipolar distance in the ovalbumin in vitro test [30]. The in vitro ovalbumin model is often used to simulate the internal environment of the human body $[15,31]$. The results of the ovalbumin in vitro test indicated a difference in the effects of radiofrequency thermocoagulation between the bipolar and unipolar groups. When the distance of the two electrodes was 5 $\mathrm{mm}$, the effects of the radio frequency thermocoagulation was optimal. It is even more surprising that when the bipolar radio frequency reached approximately 120 seconds, the coagulation volume of ovalbumin remained constant with time. It is uncertain whether the RF time can be reduced to 120 seconds for the patients in a clinical setting.
Through the above research, it was found that if the radio frequency time is prolonged as much as possible, which means increasing the degree or extent of damage, there would not be more complications or more harm to patients. In the future, in order to achieve better clinical treatment results, we will try to further increase the scope of surgical damage, in order to achieve better clinical treatment results. In the end, we want to do a single treatment which is permanently effective.

The present study exhibits certain shortcomings. First of all, it is a single-center study with relatively limited clinical samples. Secondly, we followed-up the subjects to a maximum time duration of 2 years after the operation. Therefore, we may need to increase the follow-up time to further clarify the curative effect, such as at 3 years and 5 years after the operation. Furthermore, in the ovalbumin in vitro test, we did not further explore the spatial position and temperature gradient of the electrode, such as crossover, tip adjacency, or tail adjacency in the three-dimensional space. The aforementioned shortcomings will be improved and optimized in future research.

To sum up, radiofrequency thermocoagulation of the bipolar DRG exhibited a longer duration in the treatment of PHN and the effects of radiofrequency thermocoagulation were optimal when the distance between the two electrodes was $5 \mathrm{~mm}$, which is helpful in guiding clinical work and providing more effective treatment for patients.

In summary, bipolar spinal DRG radiofrequency thermocoagulation has a long duration and high rate of effectiveness in the treatment of PHN. DCRF with a $5 \mathrm{~mm}$ spacing exhibits a longer duration and higher rate of effectiveness in the treatment of PHN. Therefore, it may be a new choice for the clinical treatment of PHN.

\section{ACKNOWLEDGMENTS}

The authors of this project are very grateful for the understanding and support of the patients and their families who participated in this study.

\section{CONFLICT OF INTEREST}

No potential conflict of interest relevant to this article was reported.

\section{FUNDING}

The present study was supported by the National Natural Science Foundation of China (81901124), Natural Science 
Foundation of Zhejiang Province of China (LY20H090020, LGF20H090021, LQ19H090007), Key Discipline Established by Zhejiang Province and Jiaxing City Jointly --Pain Medicine (2019-ss-ttyx) and Jiaxing Key Laboratory of Neurology and Pain Medicine.

\section{ORCID}

Jianjun Zhu, https://orcid.org/0000-0002-5725-7152

Ge Luo, https://orcid.org/0000-0001-6681-9308

Qiuli He, https://orcid.org/0000-0003-1339-8647

Ming Yao, https://orcid.org/0000-0002-3014-0560

\section{REFERENCES}

1. Kennedy PGE, Gershon AA. Clinical features of varicellazoster virus infection. Viruses 2018; 10: 609.

2. Freer G, Pistello M. Varicella-zoster virus infection: natural history, clinical manifestations, immunity and current and future vaccination strategies. New Microbiol 2018; 41: 95-105.

3. Kawai K, Yawn BP. Risk factors for herpes zoster: a systematic review and meta-analysis. Mayo Clin Proc 2017; 92: 1806-21.

4. Cunningham AL, Levin MJ. Herpes zoster vaccines. J Infect Dis 2018; 218(suppl_2): S127-33.

5. Schmader K. Herpes zoster. Ann Intern Med 2018; 169: ITC19-31.

6. Li JY. Herpes zoster ophthalmicus: acute keratitis. Curr Opin Ophthalmol 2018; 29: 328-33.

7. Kawai K, Yawn BP, Wollan P, Harpaz R. Increasing incidence of herpes zoster over a 60 -year period from a populationbased study. Clin Infect Dis 2016; 63: 221-6.

8. Forbes HJ, Thomas SL, Smeeth L, Clayton T, Farmer R, Bhaskaran $\mathrm{K}$, et al. A systematic review and meta-analysis of risk factors for postherpetic neuralgia. Pain 2016; 157: 30-54.

9. Koshy E, Mengting L, Kumar H, Jianbo W. Epidemiology, treatment and prevention of herpes zoster: a comprehensive review. Indian J Dermatol Venereol Leprol 2018; 84: 251-62.

10. Gross GE, Eisert L, Doerr HW, Fickenscher H, Knuf M, Maier $\mathrm{P}$, et al. S2k guidelines for the diagnosis and treatment of herpes zoster and postherpetic neuralgia. J Dtsch Dermatol Ges 2020; 18: 55-78.

11. Kim ED, Lee YI, Park HJ. Comparison of efficacy of continuous epidural block and pulsed radiofrequency to the dorsal root ganglion for management of pain persisting beyond the acute phase of herpes zoster. PLoS One 2017; 12: e0183559.

12. Kim K, Jo D, Kim E. Pulsed radiofrequency to the dorsal root ganglion in acute herpes zoster and postherpetic neuralgia. Pain Physician 2017; 20: E411-8.

13. Cahana A, Vutskits L, Muller D. Acute differential modulation of synaptic transmission and cell survival during expo- sure to pulsed and continuous radiofrequency energy. J Pain 2003; 4: 197-202.

14. Usmani H, Dureja GP, Andleeb R, Tauheed N, Asif N. Conventional radiofrequency thermocoagulation vs pulsed radiofrequency neuromodulation of ganglion Impar in chronic perineal pain of nononcological origin. Pain Med 2018; 19: 2348-56.

15. Huang B, Xie K, Chen Y, Wu J, Yao M. Bipolar radiofrequency ablation of mandibular branch for refractory V3 trigeminal neuralgia. J Pain Res 2019; 12: 1465-74.

16. Lin H, Cao G, Jin G, Yang Z, Huang C, Shao J, et al. Extracranial non-gasserian ganglion application of radiofrequency thermocoagulation on the mandibular branch of the trigeminal through the foramen ovale for trigeminal neuralgia. Pain Physician 2021; 24: E425-32.

17. Chang AK, Bijur PE, Esses D, Barnaby DP, Baer J. Effect of a single dose of oral opioid and nonopioid analgesics on acute extremity pain in the emergency department: a randomized clinical trial. JAMA 2017; 318: 1661-7.

18. Matsumoto I, Kamei K, Murase T, Yoshida Y, Kawaguchi K, Matsumoto M, et al. Surgical treatment for chronic pancreatitis: a single-center retrospective study in Japan. J Hepatobiliary Pancreat Sci 2020; 27: 632-9.

19. Issa Y, Kempeneers MA, Bruno MJ, Fockens P, Poley JW, Ahmed Ali U, et al. Effect of early surgery vs endoscopy-first approach on pain in patients with chronic pancreatitis: the ESCAPE randomized clinical trial. JAMA 2020; 323: 237-47.

20. Fang L, Jingjing L, Ying S, Lan M, Tao W, Nan J. Computerized tomography-guided sphenopalatine ganglion pulsed radiofrequency treatment in 16 patients with refractory cluster headaches: twelve- to 30-month follow-up evaluations. Cephalalgia 2016; 36: 106-12.

21. Yao P, Hong T, Wang ZB, Ma JM, Zhu YQ, Li HX, et al. Treatment of bilateral idiopathic trigeminal neuralgia by radiofrequency thermocoagulation at different temperatures. Medicine (Baltimore) 2016; 95: e4274.

22. Ding Y, Li H, Hong T, Zhu Y, Yao P, Zhou G. Combination of pulsed radiofrequency with continuous radiofrequency thermocoagulation at low temperature improves efficacy and safety in V2/V3 primary trigeminal neuralgia. Pain Physician 2018; 21: E545-53.

23. Elawamy A, Abdalla EEM, Shehata GA. Effects of pulsed versus conventional versus combined radiofrequency for the treatment of trigeminal neuralgia: a prospective study. Pain Physician 2017; 20: E873-81.

24. Song L, He L, Pei Q, Peng K, Wang N, Guo Z, et al. CT-guided percutaneous radiofrequency thermocoagulation for glossopharyngeal neuralgia: a retrospective clinical study of 117 cases. Clin Neurol Neurosurg 2019; 178: 42-5.

25. Ding Y, Yao P, Li H, Han Z, Wang S, Hong T, et al. CT-guided stellate ganglion pulsed radiofrequency stimulation for facial and upper limb postherpetic neuralgia. Front Neurosci 
2019; 13: 170.

26. Lin CS, Lin YC, Lao HC, Chen CC. Interventional treatments for postherpetic neuralgia: a systematic review. Pain Physician 2019; 22: 209-28.

27. Gulec E, Ozbek H, Pektas S, Isik G. Bipolar versus unipolar intraarticular pulsed radiofrequency thermocoagulation in chronic knee pain treatment: a prospective randomized trial. Pain Physician 2017; 20: 197-206.

28. Wan C, Dong DS, Song T. High-voltage, long-duration pulsed radiofrequency on Gasserian ganglion improves acute/subacute zoster-related trigeminal neuralgia: a randomized, double-blinded, controlled trial. Pain Physician 2019; 22: 361-8.
29. Arakawa K, Kaku R, Kurita M, Matsuoka Y, Morimatsu H. Prolonged-duration pulsed radiofrequency is associated with increased neuronal damage without further antiallodynic effects in neuropathic pain model rats. J Pain Res 2018; 11: 2645-51.

30. Zhang C, Wang P, Liu A, Zhang J, Yin G, Jin Z, et al. [Doseeffect relationships of bipolar radio frequency heating]. Chin J Phys Med Rehabil 2018; 40: 414-7. Chinese.

31. Luo F, Yu XT, Li DY, Huang YQ, Wu DS, Cui JJ, et al. [The safety of the frequency and temperature parameters of pulsed radiofrequency by egg white experiment]. Chin J Pain Med 2012; 18: 742-5. Chinese. 